\title{
Weight gain since menopause and its associations with weight loss maintenance in obese postmenopausal women
}

\author{
M Sénéchal ${ }^{1,2}$ \\ H Arguin ${ }^{6}$ \\ DR Bouchard 4,5 \\ AC Carpentier ${ }^{3}$ \\ JL Ardilouze ${ }^{3}$ \\ IJ Dionne ${ }^{1,2}$ \\ M Brochu ${ }^{1,2}$
}

\begin{abstract}
'Research Centre on Aging, Health and Social Services Centre, University Institute of Geriatrics of Sherbrooke, ${ }^{2}$ Faculty of Physical Education and Sports, University of Sherbrooke, ${ }^{3}$ Clinical Research Centre, Sherbrooke University Hospital Centre, Sherbrooke, QC; ${ }^{4}$ Faculty of Kinesiology and Recreation Management, University of Manitoba, Winnipeg, MB; ${ }^{5}$ Health, Leisure \& Human Performance Research Institute, Winnipeg, MB; ${ }^{6}$ Division of Kinesiology, Department of Social and Preventive Medicine, Faculty of Medicine, Laval University, QC, Canada
\end{abstract}

Correspondence: Martin Brochu Research Centre on Aging, 1036 Belvédère Sud, Sherbrooke, QC, Canada JIH 4C4

Tel + I 8197802220 ext 45326

Fax $+|819829714|$

Email martin.brochu@usherbrooke.ca
This article was published in the following Dove Press journal:

Clinical Interventions in Aging

18 August 2011

Number of times this article has been viewed
Objective: To examine the association between weight gain since menopause and weight regain after a weight loss program.

Methods: Participants were 19 obese women who participated in a 15 -week weight loss program and a 12-month follow-up. Main outcomes were: body composition, resting metabolic rate, energy intake, energy expenditure, and weight regain at follow-up.

Results: All body composition measures significantly decreased after intervention (all $P \leq 0.01$ ) while all measures of fatness increased significantly after the 12-month follow-up $(P \leq 0.01)$. Body weight gain since menopause was associated with body weight regain $(r=0.65 ; P=0.003)$ after follow-up even after adjustment for confounders.

Conclusion: Weight gain since menopause is associated with body weight regain following the weight loss program. Therefore, weight gain since menopause should be considered as a factor influencing weight loss maintenance in older women.

Keywords: obesity, body weight, weight regain, postmenopausal women, aging

\section{Introduction}

The prevalence of obesity has increased significantly over the past few decades with the highest prevalence being in women aged between 45 and 64 years. ${ }^{1}$ As previously reported, increase in body weight is associated with increased risks of metabolic disorders such as metabolic syndrome, type 2 diabetes, and cardiovascular diseases. ${ }^{2}$

The beneficial effects of caloric restriction-induced weight loss on body composition and health are well established. ${ }^{3}$ A study by Weiss et $a l^{4}$ revealed that more than $50 \%$ of women try to lose weight after menopause. Unfortunately, most of them will regain weight loss and even more over the following years, ${ }^{5}$ dampening the health benefits associated with weight loss. Studies have reported many factors associated with body weight regain following a weight loss program, including physiologic, ${ }^{6}$ metabolic, ${ }^{7}$ psychological, ${ }^{8}$ and behavioral ${ }^{9}$ factors.

Lifetime body weight has been associated with higher risk of cardiovascular diseases,${ }^{10}$ cancer, ${ }^{11}$ and weight regain following a weight loss intervention in young women. ${ }^{12}$ However, this issue has yet received little attention in postmenopausal women. ${ }^{13}$ Investigating the association between body weight gain since menopause and weight loss maintenance is of great interest considering that peak body weight gain is observed around age 50 in women, ${ }^{14}$ weight gain rate is also high during this period, ${ }^{15}$ and successful weight maintenance remains a challenge in all age groups. ${ }^{5}$ Furthermore, weight gain since menopause may help identify older women at higher risk of body weight regain after weight loss programs. ${ }^{8}$ Thus, the present study was 
conducted to examine the association between weight gain since menopause and weight regain after a weight loss program in postmenopausal women.

\section{Methods}

\section{Population}

Twenty-five postmenopausal women volunteered, and were recruited by advertising in the general community (eg, local journal). From those, six did not complete the study because they did not follow the weight loss protocol. Consequently, 19 obese postmenopausal women aged between 50 and 75 years participated in the study. Inclusion criteria: no menstruation in a previous year, obese ( $\geq 35 \%$ body fat), ${ }^{16,17}$ waist circumference $\geq 90 \mathrm{~cm}$, sedentary ( $<$ two times a week of structured exercise), nonsmokers, low alcohol consumers ( $<$ two drinks per day), and had no body weight fluctuation $\geq 5 \mathrm{~kg}$ in the 6 months prior to the study. On physical examination and biological testing, all participants had no history or evidence of cardiovascular disease, peripheral vascular disease, stroke, or diabetes (fasting glucose $\geq 7.0 \mathrm{mmol} / \mathrm{L}$ ). Moreover, all participants did not use hormonal replacement therapy in the 6 months prior to the study and were not taking any medication that could affect blood pressure, lipids and lipoproteins, glucose homeostasis, thyroid, and resting metabolic rate (RMR). All participants signed an informed consent document from the ethics committees of the Health and Social Services, University Institute of Geriatrics of Sherbrooke and Sherbrooke University Hospital Centre.

\section{Caloric restriction intervention}

Dietary intervention was designed to reduce body weight by $1 \%$ of initial body weight per week for a maximum of 15 weeks. Prior to baseline testing, a period of body weight stabilization was executed ( $\pm 2 \mathrm{~kg}$ during 4 consecutive weeks). A food diary was used during the weight stabilization period to help subjects monitor their food intake and to quantify total daily caloric intake. The mean daily intake recorded during the last 7 days prior to study was used to determine baseline total daily caloric intakes. The energy deficit needed to achieve the amount of weight loss per week was then determined for each participant by subtracting the energy equivalent to $1 \%$ of initial body weight to the total daily caloric intake. Food was self-selected under dietician supervision but a standard diet was followed with $55 \%$ of carbohydrates, $30 \%$ of fat, and $15 \%$ of energy intake from proteins. ${ }^{18}$

Subjects were asked to bring their food diary to every weekly group session on nutrition information (45 minutes) given by a dietician. Results were closely monitored and the dietician individually supervised the following week caloric goal.

\section{Energy intake}

Subjects were provided with a food scale and instructed on how to complete a 3-day dietary record. Caloric intake was calculated using a 3-day food diary (2 week days and 1 weekend day). Daily caloric intake was calculated using Candat System software (v 6.0; Godin London Inc, London, ON).

\section{Energy expenditure}

Daily energy expenditure was estimated using a Caltrac uniaxial accelerometer (Muscle Dynamics Fitness Network, Torrance, CA). The accelerometer was attached to the waist and worn during all waking hours over a 3-day period (2 week days and 1 day over the weekend). ${ }^{19}$ Average kcal/ day over the measurement period was used for data analyses. Participants were asked not to enroll in an exercise program and to maintain their baseline physical activity throughout the duration of the study.

\section{Anthropometric and body composition measures}

Body weight was measured to the nearest $0.2 \mathrm{~kg}$ on a calibrated balance (Seca 707; Seca, Hamburg, Germany) and height was obtained with a standard stadiometer (Takei Scientific Instruments Co Ltd, Tokyo, Japan). Waist circumference was measured with a steel measuring tape to the nearest $0.1 \mathrm{~cm}$ at the highest point of the iliac crest at minimal respiration. Body composition was assessed using dual emission X-ray absorptiometry (GE Lunar Corp, Madison, WI), as previously described. ${ }^{20}$ Finally, weight gain since menopause was calculated by using self-reported body weight gain since having no menstruation for a complete year or menopause diagnosis by measuring follicle stimulating hormone level. Despite relying on a self-reported body weight gain since menopause, it has been previously shown that self-reported body weight is a reliable measure specifically in older adults (ranging $r=0.85-0.98) .{ }^{21-23}$

\section{RMR}

The test was done in the morning after a 12-hour fasting period. Subjects were asked to consume no alcohol, not to take any medication, and to restrain physical activity for 24 hours before testing. RMR was measured on a 30-minute period by indirect calorimetry. RMR (kcal/day) was calculated using the Weir equation. $\mathrm{VO}_{2}$ and $\mathrm{VCO}_{2}$ were measured using a CCM/D metabolic cart (Medical Graphics 
Corporation, St Paul, MN) and resting respiratory quotient. Both absolute and relative RMR (RMR/lean body mass) were used in analyses.

\section{Statistical analyses}

Normality of variables was tested by Shapiro-Wilk tests. Wilcoxon signed rank tests were performed to quantify the effect of treatment. Spearman's correlations were used to quantify the relationship between body weight since menopause and body weight regain after the 12-month follow-up. Finally, partial correlation was used to assess the correlation between body weight since menopause and body weight regain after the 12-month follow-up adjusted for potential confounders. Variables not normally distributed were log transformed to normalize their distribution before performing partial correlation. Analyses were performed using SPSS (v 18.0; SPSS Inc, Chicago, IL). A level of significance of $P \leq 0.05$ was used unless stated otherwise.

\section{Results}

Women enrolled in this study were $12 \pm 9$ years since menopause, while the average weight gain since menopause was $10.7 \pm 5.6 \mathrm{~kg}$ (Table 1 ). As expected, energy intake decreased significantly during the intervention (mean $-354 \pm 443 \mathrm{kcal} / \mathrm{day} ; P<0.01)$ especially from carbohydrate and fat (mean $-38.8 \pm 70.9 \mathrm{~g} /$ day, $-19.2 \pm 24.1 \mathrm{~g} /$ day; both $P<0.05$ ). Moreover, all fat types (trans, saturated, monounsaturated, and polyunsaturated fat) significantly decreased during the intervention (all $P<0.05)$. A significant decrease was also observed for all adiposity measures (body weight, body mass index, waist circumference, and fat mass) after the 15-week intervention (all $P<0.01$ ). Finally, lean body mass also significantly decreased during the intervention $(P<0.01)$, while RMR and daily energy expenditure remained stable.

On average, women regained $2.5 \pm 3.3 \mathrm{~kg}$ body weight at 12-month follow-up $(P<0.01)$ and increased body mass index, waist circumference, and fat mass (all $P<0.01$ ). No significant change was observed for lean body mass, RMR, and energy intake at follow-up. Finally, energy expenditure significantly decreased during 12-month follow-up $(P<0.01)$.

Weight gain since menopause $(r=0.65 ; P=0.003)$ was associated with weight regain at follow-up. However, baseline body weight ( $r=0.48 ; P=0.03)$, body mass index $(r=0.58 ; P=0.008)$, fat mass $(r=0.44 ; P=0.05)$, absolute $\mathrm{RMR}(r=0.65 ; P=0.002)$, and relative $\mathrm{RMR}$

Table I Characteristics of the cohort

\begin{tabular}{|c|c|c|c|}
\hline$N=19$ & Baseline & Post- intervention & I 2-month follow-up \\
\hline Age (years) & $61.2 \pm 6.0$ & - & - \\
\hline Age at menopause (years) & $49.2 \pm 7.1$ & - & - \\
\hline Years since menopause (years) & $12.0 \pm 8.8$ & - & - \\
\hline Body weight gain since menopause (kg) & $10.7 \pm 5.6$ & - & - \\
\hline Body weight (kg) & $79.3 \pm 11.1$ & $69.2 \pm 10.2^{*}$ & $71.8 \pm 12.4^{\ddagger}$ \\
\hline BMI $\left(\mathrm{kg} / \mathrm{m}^{2}\right)$ & $31.8 \pm 4.0$ & $27.5 \pm 3.8^{*}$ & $28.7 \pm 4.9^{\ddagger}$ \\
\hline Waist circumference $(\mathrm{cm})$ & $98.6 \pm 8.7$ & $88.0 \pm 7.4^{*}$ & $93.1 \pm 10.0^{\ddagger}$ \\
\hline Total fat mass (kg) & $36.7 \pm 8.4$ & $27.8 \pm 8.1^{*}$ & $30.5 \pm 9.4^{\ddagger}$ \\
\hline Trunk fat mass (kg) & $18.6 \pm 3.9$ & $12.9 \pm 3.7^{*}$ & $14.0 \pm 4.5^{\ddagger}$ \\
\hline Appendicular fat mass (kg) & $17.1 \pm 4.6$ & $14.1 \pm 4.4^{*}$ & $15.6 \pm 5.1^{\ddagger}$ \\
\hline Total lean body mass (kg) & $40.3 \pm 4.5$ & $39.1 \pm 3.8^{*}$ & $39.0 \pm 4.1$ \\
\hline Absolute RMR (kcal/day) & $1,170 \pm 186$ & $1,209 \pm 198$ & $1,270 \pm 192$ \\
\hline Relative RMR (kcal/kg of lean body mass) & $29.1 \pm 3.9$ & $30.8 \pm 3.9$ & $32.5 \pm 3.7$ \\
\hline Energy intake (kcal/day) & $1,875 \pm 490$ & $1,539 \pm 340 *$ & $1,410 \pm 277$ \\
\hline Total carbohydrates (g) & $236.5 \pm 69.2$ & $199.7 \pm 43.3^{*}$ & $175.0 \pm 32.4$ \\
\hline Total proteins (g) & $86.0 \pm 21.3$ & $79.1 \pm 20.9$ & $70.9 \pm 13.0$ \\
\hline Total fat (g) & $66.6 \pm 23.2$ & $47.5 \pm 18.5^{*}$ & $45.0 \pm 14.3$ \\
\hline Trans fat (g) & $0.49 \pm 0.46$ & $0.38 \pm 0.88^{*}$ & $0.52 \pm 1.0$ \\
\hline Saturated fat (g) & $19.9 \pm 9.5$ & $14.2 \pm 6.6^{*}$ & $12.1 \pm 5.2$ \\
\hline Monounsaturated fat (g) & $24.6 \pm 9.6$ & $14.7 \pm 6.2^{*}$ & $15.4 \pm 6.2$ \\
\hline Polyunsaturated fat (g) & $12.8 \pm 6.5$ & $8.3 \pm 3.6^{*}$ & $8.7 \pm 3.5$ \\
\hline Estimated daily energy expenditure (kcal/day) & $2,084 \pm 448$ & $1,956 \pm 426$ & $1,747 \pm 304^{\ddagger}$ \\
\hline
\end{tabular}

Notes: Data are presented as means \pm SD. Wilcoxon signed rank tests were used to measure the intervention effect between baseline and post-intervention and postintervention and I2-month follow-up. *Significant difference between post-intervention and baseline values, $P<0.05$; $¥$ significant difference between 12 -month follow-up and post-intervention values, $P<0.05$.

Abbreviations: BMI, body mass index; RMR, resting metabolic rate. 
Table 2 Correlations between independent variables of interest and body weight maintenance at I2-month follow-up

\begin{tabular}{ll}
\hline & Spearman's correlations \\
\cline { 2 - 2 } Variable & Body weight regain \\
\hline Age & $0.13(0.570)$ \\
Age at menopause & $-0.34(0.140)$ \\
Body weight gain since menopause & $0.65(0.003)$ \\
Baseline body weight & $0.48(0.030)$ \\
BMI & $0.61(0.005)$ \\
Waist circumference & $0.15(0.530)$ \\
Total fat mass & $0.44(0.050)$ \\
Trunk fat mass & $0.43(0.060)$ \\
Appendicular fat mass & $0.43(0.060)$ \\
Total lean body mass & $0.35(0.140)$ \\
Absolute RMR & $0.65(0.002)$ \\
Relative RMR & $0.48(0.030)$ \\
Energy intake & $-0.02(0.910)$ \\
Estimated daily energy expenditure & $0.39(0.090)$ \\
\hline
\end{tabular}

Notes: Results are coefficients of correlation ( $P$ value). $\Delta$ Body weight at I2-month follow-up = changes between 12-month follow-up and post-intervention.

Abbreviations: BMI, body mass index; RMR, resting metabolic rate.

( $r=0.49 ; P=0.03$ ) were also correlated with weight regain after the 12-month follow-up (Table 2). Nonetheless, weight gain since menopause remained significantly associated with weight regain at follow-up once adjusted for years since menopause and initial body weight $(r=0.50 ; P=0.03)$ (results not shown).

\section{Discussion}

The current study suggests that women who gained more weight since menopause are at greater risk to be unsuccessful in their weight loss attempt (with caloric restriction) within a few years after intervention. Therefore, weight gain during the menopause period has to be monitored closely because it may be hard to successfully lose those extra pounds and keep them off after menopause.

The fact that initial fat mass, lean body mass, and RMR were associated with body weight regain is not novel. ${ }^{6}$ However, with this study we add another variable that needs to be taken into account when evaluating the risk of weight regain following a caloric restriction in postmenopausal women. Several studies have reported an association between loss in lean body mass and decrease in RMR after weight loss. ${ }^{24}$ Surprisingly, we observed no overall decrease in RMR after the intervention despite significant decreases in lean body mass. It is likely that the mean lean body mass loss of $1.3 \pm 1.3 \mathrm{~kg}$ or $3.2 \%$ in the present study might be statistically significant but clinically trivial. In fact, Busetto et a ${ }^{25}$ reported a mean lean body mass loss of $9.4 \%$, while we only observed a mean loss of $3.2 \%$. Consequently, the observed difference between study results could be explained by the magnitude decrease in lean body mass during weight loss intervention.

From a clinical point of view, the fact that weight gain since menopause might be an important component of weight regain after a caloric restriction intervention is of great interest considering that more than $50 \%$ of women in the menopausal period try to lose weight without successful maintenance. ${ }^{4}$ Determining how much weight was gained since menopause, as one of the variables that predicts weight loss maintenance, could be useful since that information is easy to get. A question asking women how much weight they gained since menopause may be too simple and invalid but a study conducted by Lawlor et $\mathrm{a}^{22}$ concluded that selfreported and measured body weight are highly correlated in older adults $(r=0.98)$. Thus, women who report gaining a large amount of weight since menopause should have a tight follow-up to favor weight loss maintenance. In 2005, the Obesity Society and the American Society for Nutrition stated that innovative studies are needed to identify and better understand factors associated with weight loss maintenance in older individual ${ }^{24}$ and this study, with some reservations, goes exactly in that direction.

As this study was exploratory and based on a small sample, many limitations of this study must be mentioned. First, the lack of a control group and the small sample size affect the external validity of the results. Consequently, results translate only to obese postmenopausal women aged between 50-75 years. Second, weight gain since menopause was evaluated with a single question. Thus, objective measure of lifetime body weight would be needed to validate the results. Moreover, we do not have the obesity status before menopause which could impact on the association between weight gain since menopause and weight regain. Similarly, some may argue that age of menopause can also play a role in this association. However, we can partially rule out this hypothesis since we observed no significant correlation between weight loss maintenance and the duration of menopausal status $(r=0.37 ; P=0.11)$. Third, because of the study design, it is not possible to investigate mechanisms or other variables that may help to understand our results. Despite these limitations, we need to highlight that these results are based on a well-characterized sample of obese postmenopausal women and the limited number of subjects allowed us to closely follow the participants. Nonetheless, our results are really intriguing and need to be confirmed with a tighter design and objective methods to assess the exact time of menopause as well as a measured follow-up of body weight since menopause. 
In conclusion, our study suggests that greater weight gain since menopause is correlated with lower weight maintenance after a caloric restriction intervention in obese postmenopausal women. Indeed, weight gain since menopause could be considered a variable to identify postmenopausal obese women at greater risk of weight regain after a caloric restriction regimen. Despite this intriguing finding, further studies are needed to validate our results.

\section{Acknowledgments}

This work was supported by the Canadian Institute of Health Research (MS, DRB, and IJD), and was performed in part at the Centre de recherché Clinique Étienne-Le Bel and the Centre de recherche sur le vieillissement de Sherbrooke, both centers funded by the Fonds de la Recherche en Santé du Québec. We would also like to thank the research nurses.

\section{Disclosure}

The authors report no conflicts of interest in this work.

\section{References}

1. Tjepkema M. Adult obesity in Canada: measured height and weight. 2005. Available from: http://www.statcan.gc.ca/pub/82-620-m/2005001/ article/adults-adultes/8060-eng.htm. Accessed June 1, 2011.

2. Lemay A, Turcot L, Dechene F, Dodin S, Forest JC. Hyperinsulinemia in nonobese women reporting a moderate weight gain at the beginning of menopause: a useful early measure of susceptibility to insulin resistance. Menopause. 2010;17(2):321-325.

3. Chaston TB, Dixon JB. Factors associated with percent change in visceral versus subcutaneous abdominal fat during weight loss: findings from a systematic review. Int J Obes (Lond). 2008;32(4):619-628.

4. Weiss EC, Galuska DA, Khan LK, Serdula MK. Weight-control practices among US adults, 2001-2002. Am J Prev Med. 2006;31(1):18-24.

5. Mann T, Tomiyama AJ, Westling E, Lew AM, Samuels B, Chatman J. Medicare's search for effective obesity treatments: diets are not the answer. Am Psychol. 2007;62(3):220-233.

6. Vogels N, Diepvens K, Westerterp-Plantenga MS. Predictors of longterm weight maintenance. Obes Res. 2005;13(12):2162-2168

7. Boule NG, Chaput JP, Doucet E, et al. Glucose homeostasis predicts weight gain: prospective and clinical evidence. Diabetes Metab Res Rev. 2008;24(2):123-129.

8. Teixeira PJ, Going SB, Sardinha LB, Lohman TG. A review of psychosocial pre-treatment predictors of weight control. Obes Rev. 2005;6(1):43-65.

9. Wing RR, Phelan S. Long-term weight loss maintenance. Am J Clin Nutr. 2005;82(1 Suppl):222S-225S

10. Elfhag K, Rossner S. Who succeeds in maintaining weight loss? A conceptual review of factors associated with weight loss maintenance and weight regain. Obes Rev. 2005;6(1):67-85.

Clinical Interventions in Aging

\section{Publish your work in this journal}

Clinical Interventions in Aging is an international, peer-reviewed journal focusing on evidence-based reports on the value or lack thereof of treatments intended to prevent or delay the onset of maladaptive correlates of aging in human beings. This journal is indexed on PubMed Central, MedLine, the American Chemical Society's 'Chemical
11. Eliassen AH, Colditz GA, Rosner B, Willett WC, Hankinson SE. Adult weight change and risk of postmenopausal breast cancer. JAMA. 2006;296(2):193-201.

12. Field AE, Manson JE, Taylor CB, Willett WC, Colditz GA. Association of weight change, weight control practices, and weight cycling among women in the Nurses' Health Study II. Int J Obes Relat Metab Disord. 2004;28(9):1134-1142.

13. Teixeira PJ, Palmeira AL, Branco TL, et al. Who will lose weight? A reexamination of predictors of weight loss in women. Int J Behav Nutr Phys Act. 2004;1(1):12.

14. Guo SS, Zeller C, Chumlea WC, Siervogel RM. Aging, body composition, and lifestyle: the Fels Longitudinal Study. Am J Clin Nutr. 1999;70(3):405-411.

15. Lewis CE, Smith DE, Wallace DD, Williams OD, Bild DE, Jacobs DR Jr. Seven-year trends in body weight and associations with lifestyle and behavioral characteristics in black and white young adults: the CARDIA study. Am J Public Health. 1997;87(4):635-642.

16. Durstine JL, King AC, Painter PL, Roitman JL, Zwiren LID, Kenney WL, editors. ACSM's Resource Manual for Guidelines for Exercise Testing and Prescription. 2nd edition. Philadelphia, PA: Lea and Ferbiger; 1993.

17. Aubertin-Leheudre M, Lord C, Goulet ED, Khalil A, Dionne IJ. Effect of sarcopenia on cardiovascular disease risk factors in obese postmenopausal women. Obesity (Silver Spring). 2006;14(12):2277-2283.

18. Krauss RM, Eckel RH, Howard B, et al. AHA Dietary Guidelines: revision 2000: a statement for healthcare professionals from the Nutrition Committee of the American Heart Association. Stroke. 2000;31(11):2751-2766.

19. Wong TC, Webster JG, Montoye HJ, Washburn R. Portable accelerometer device for measuring human energy expenditure. IEEE Trans Biomed Eng. 1981;28(6):467-471.

20. Arguin H, Bouchard DR, Labonte M, et al. Correlation between the rate of weight loss and changes in body composition in obese postmenopausal women after 5 weeks: a pilot study. Appl Physiol Nutr Metab. 2008;33(2):347-355.

21. Stevens J, Keil JE, Waid LR, Gazes PC. Accuracy of current, 4-year, and 28-year self-reported body weight in an elderly population. $\mathrm{Am} J$ Epidemiol. 1990;132(6):1156-1163.

22. Lawlor DA, Bedford C, Taylor M, Ebrahim S. Agreement between measured and self-reported weight in older women. Results from the British Women's Heart and Health Study. Age Ageing. 2002;31(3):169-174.

23. Fillenbaum GG, Kuchibhatla MN, Whitson HE, et al. Accuracy of self-reported height and weight in a community-based sample of older African Americans and whites. J Gerontol A Biol Sci Med Sci. 2010;65(10):1123-1129.

24. Villareal DT, Apovian CM, Kushner RF, Klein S. Obesity in older adults: technical review and position statement of the American Society for Nutrition and NAASO, The Obesity Society. Obes Res. 2005;13(11):1849-1863.

25. Busetto L, Perini P, Giantin V, et al. Relationship between energy expenditure and visceral fat accumulation in obese women submitted to adjustable silicone gastric banding (ASGB). Int J Obes Relat Metab Disord. 1995;19(4):227-233.

Abstracts Service' (CAS), Scopus and the Elsevier Bibliographic databases. The manuscript management system is completely online and includes a very quick and fair peer-review system, which is all easy to use. Visit http://www.dovepress.com/testimonials.php to read real quotes from published authors. 